\title{
Deep Drawing with Local Hardening on Digital Multi-axis Servo Press
}

\author{
Sebastian Kriechenbauer ${ }^{1} \cdot$ Reinhard Mauermann $^{1} \cdot$ Dirk Landgrebe $^{1}$
}

Received: 29 June 2015/Revised: 6 November 2015/Published online: 9 December 2015

(C) The Chinese Society for Metals and Springer-Verlag Berlin Heidelberg 2015

\begin{abstract}
The paper discusses a new drawing technology, based on a synchronized movement of ram and cushion with multiple bending operations in alternating directions called "bi-directional deep drawing (BDD)." The goal is to avoid local thinning by strengthening the weak point using local hardening. BDD operations are realized before the conventional deep drawing process. This results in a local strain hardening at the weak point of the workpiece, which is usually located at the bottom punch radius. Two major aspects have to be given attention due to the high number of process parameters. On the one hand, for process design, it is helpful to have a tool by means of which it is possible to simultaneously create both the machine program for the servo press and the initial configuration for the process simulation. From the authors' point of view, this complexity can only be represented by a numerical analysis method, on the other hand. Consequently, both aspects are given attention in this paper.
\end{abstract}

KEY WORDS: Bi-directional deep drawing (BDD); Local hardening; Servo press; Pulsation

\section{Introduction}

Today's servo presses achieve productivity gains mainly by adjusting the velocity profiles in the non-piece-contact ram movement. Influencing the drawing process by kinematic adjustment during workpiece contact is made possible by the new dynamic characteristics of servo presses. These developments are still at the beginning.

Current research projects explore the modification of the strain rate during forming. One objective, for example, is to affect the tribologic conditions in the interaction of the sheet and the die [1, 2]. The material behavior at the moment when the stroke is interrupted [3, 4] or the

Available online at http://link.springer.com/journal/40195

Sebastian Kriechenbauer

sebastian.kriechenbauer@iwu.fraunhofer.de

1 Fraunhofer IWU, Nöthnitzer Str. 44, 01187 Dresden, Germany deformation caused by alternating tension-compression is also examined [5]. Numerous publications reported on the use of state-of-the-art servo presses that use different design types and have modified kinematic structure [5-11].

Position-controlled path and time curves that are freely programmable have already become state of the art in hydraulic presses equipped with servo valves or advanced servo pumps. In these presses, modified kinematic structures are also implemented with vibration superposition [2, $3,5,12]$. Digital servo presses based on screw axes provide similar flexibility with greater stiffness [13].

Rapid changes in the moving direction of ram and cushion are possible (i.e., up and down) since the state-ofthe-art servo presses with higher acceleration and jerk values ensure quicker changes in velocity. There are applications for the superposition of pulsation in deep drawing processes $[3,9,14]$. In this case, primarily the ram is moved by special kinematics, and the cushion performs a synchronized movement. The limits of deep drawing may be extended by a multi-axis servo press. In cushion-ram pulsation (CRP), the drawing force is reduced by 
decoupling the forming operation (by the ram) and reduction of wrinkles (by the cushion) [9]. These tasks are performed sequentially with varying amplitudes and frequencies. The technology can be categorized as a kind of incremental forming. The drawing force required is reduced through targeted removal of stress at the flange zones for better continued flowing of the material [9]. The method is more suitable for materials with shallow flow curves, such as mild steel or aluminum.

The Bi-directional deep drawing (BDD) technology introduced here is also implemented on multi-axis servo presses.

\section{Bi-directional Deep Drawing}

In the stressed skin construction used in the automotive industry, design for high-strength steel materials is subject to stringent limits. For these materials, the individual drawn parts have significantly less complex than for conventional drawing qualities. The BDD technology was tested in order to determine the feasibility of forming highstrength sheet parts with complex geometry.

Deep drawing is characterized by specific weak points with local drawing instabilities. That is the area in the frame in which the lowest strain hardening and the highest sheet thinning are experienced. For a cylindrical cup, the critical region is the bottom radius. This zone was the first in which the structural strain appearing during drawing resulted in necking. In the BDD procedure, this zone is preliminarily locally strain-hardened by multiple alternating bending before the intrinsic deep drawing process. Thus, the potential weakness is reduced, and a higher drawing force can be transmitted to produce deeper parts at higher drawing ratios in the subsequent process.

Figure 1 illustrates the principle view of the kinematic state before conducting the deep drawing process and the die setup. Apart from the static lower drawing punch, the digital controlled axis of die and blankholder, a fixed upper punch is needed to complete the tool. The upper punch fixes only the bottom of the drawn part during reverse BDD operation.

It is possible to move all of the press axes in a positioncontrolled manner. This is the easiest solution, although it is necessary to consider the stiffness values of both the machine and the die. The forces appearing in each case are recorded in the experiment, and the resulting strains are corrected in the axis movements during iteration loops. There is another possible solution, in which one of the moved axes is forcecontrolled. Figure 2 shows complex path and force versus time curves consisting of force-controlled deep drawing and BDD operations. The table on top of the figure includes the drawing depth and cushion force of each stage. The beginning and the end of each stage are shaded in the figure. The first deep drawing stage up to 4-mm drawing depth was applied with the $100-\mathrm{kN}$ cushion force to pre-strain the material. In the second BDD stage, 6 cycles with cushion force of $50 \mathrm{kN}$ and amplitudes of 1,2 and $3 \mathrm{~mm}$ were used to reach a higher strain level at the previous drawing depth. The stage was followed by deep drawing up to further stroke of $4 \mathrm{~mm}$ and cushion force of $50 \mathrm{kN}$. The operations are repeated up to $12-\mathrm{mm}$ drawing depth. The process is completed by deep drawing with cushion force of $500 \mathrm{kN}$. Although this operation is complex, it is in general feasible within a state-of-the-art servo press control.

\section{Experiments}

The 1-mm-thick sheets made of stainless steel (1.4301) were subjected to comparative tests for the symmetrical cross-shaped cup on a digital servo press (see Fig. 3). The constraints, such as sheet blank, cushion force, velocity and oiling of the bottom, were kept constant for the comparison. During deep drawing, the typical crack appeared at less than 50-mm drawing depth. The cushion force of about approximately $100 \mathrm{kN}$ was applied to the first $12 \mathrm{~mm}$ of the drawn path, and about $500 \mathrm{kN}$ for the path up to
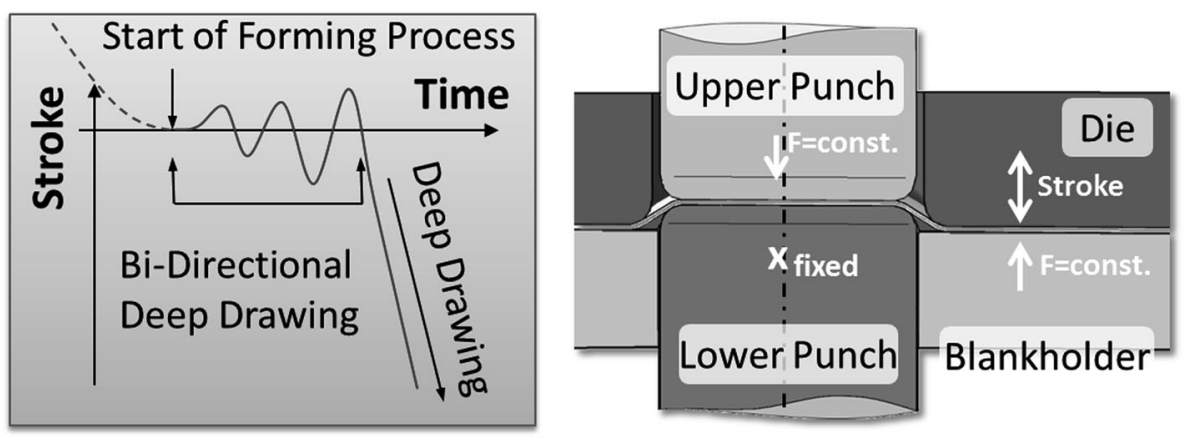

Fig. 1 Principle view of the kinematic state before conducting the deep drawing process (left) and the die setup (right) 


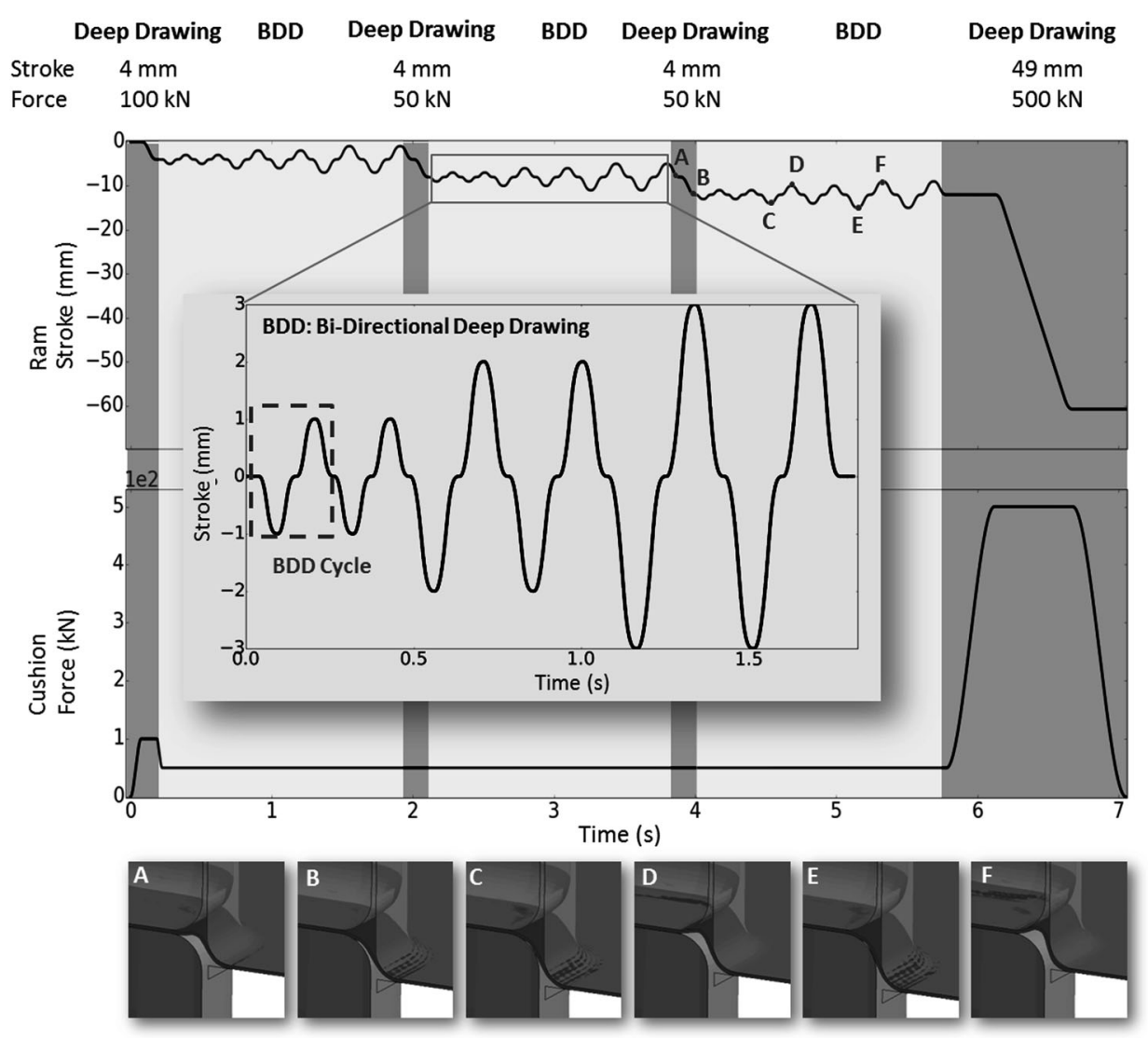

Fig. 2 Calculated path-time curve (top and middle diagram) and force-time curve (bottom diagram) for the overall process and the selected individual deformation zone views according to the process sequence

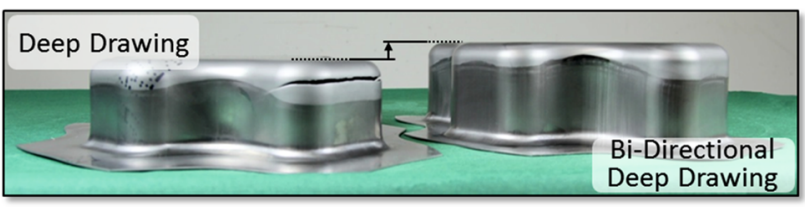

Fig. 3 Applied drawing depths in comparison: standard deep drawing procedure at minimal cushion force (left, cracked at $50-\mathrm{mm}$ drawing depth) versus BDD process (right, undamaged even at 60-mm drawing depth)

$50 \mathrm{~mm}$. In the part previously strain-hardened by means of alternating bending during BDD operation, necking could be avoided up to 60-mm drawing depth (Fig. 3). Nevertheless, the new process takes $5 \mathrm{~s}$ longer than the conventional deep drawing process, which takes only $2 \mathrm{~s}$.

A hybrid kinematic sequence (see Fig. 2) was chosen in order to homogeneously distribute the local strain hardening across the critical zone. The amplitudes of the kinematic sequence were adapted to the geometric features of the counter punch (radius $10 \mathrm{~mm}$ ), the drawing punch (bottom radius $10 \mathrm{~mm}$ ) and the drawing radius $(7.5 \mathrm{~mm}$ ). Further dimensions of the cross-shaped cup are described later on (see Table 2). Detailed technical data of used servo press could be found in Ref. [9]. During forming, the deep drawing and BDD stages alternate repeatedly. The alternating bending procedure is conducted in 18 cycles. A single cycle includes four start-stop movements (once down and back and once up and back; amplitude of plus and minus is $1 \mathrm{~mm}$, as shown in Fig. 2). In every second cycle, the amplitude of alternating bending was increased by the amount of the sheet thickness. The center was offset in three short deep drawing stages by $4 \mathrm{~mm}$ each time.

The BDD process is defined by many parameters. In each cycle, the kinematic structure can have variable path amplitudes, center offsets and force amplitudes (Fig. 2). To work efficiently, we need a program to generate the modified kinematic sequences that creates both the machine program for the servo press from the technological parameters and, simultaneously, the initial configuration for the process simulation.

\section{Kinematic Path Calculator}

The more flexible the procedure, the more complex the process setting is. In deep drawing, parameters such as the velocity and the blankholder force are predefined, whereas, in the BDD process, the number of parameters is 
significantly higher. For an efficient experimental and numeric analysis, it is necessary to process the variable technological parameters in an automated way. For this purpose, a software was built in which the user can create two data records (path and force vs. time curves) for different combinations of deep drawing processes. The software exports a start file for the simulation environment and a parameter set for the servo press.

The four start-stop movements mentioned above are each composed of a start-up procedure, travel at constant velocity and a deceleration phase. Thus one start-stop movement can be subdivided into seven segments, in which the corresponding limits for the jerk, the acceleration and the velocity have to be maintained. The shortest possible time in which the desired kinematic sequence can be implemented is calculated based on these permissible maximal values of the press axes. It is necessary to determine these limits as constants for each new servo press and to specify them in the program; in this case, for instance, the jerk limit for the ram drive is $30 \mathrm{~m} / \mathrm{s}^{3}$. The kinematic sequence providing the highest productivity, in turn, results from the technological constraints, such as amplitudes of ram and cushion, phase- and center offsets, each differing from cycle to cycle.

The graphic user interface and a plot of results that are possible in general for the BDD and CRP techniques are shown in Fig. 4. Consequently, the user is capable of flexibly configuring the kinematic sequence according to technological aspects.

\section{Process Simulation}

Given its extreme complexity, it is almost impossible to refine the BDD process in experiments by means of the conventional trial-and-error approach. For this reason, it is important to have the option of a numeric forecast. The authors carried out a first step toward the generation of a

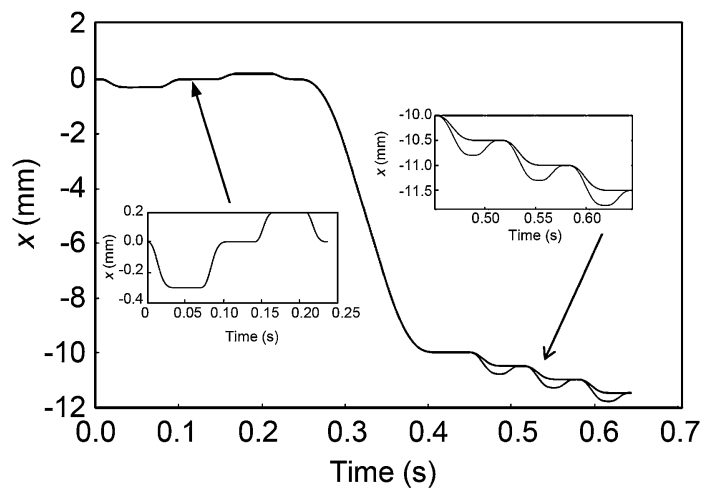

Fig. 4 Examples of a generated kinematic sequence for BDD (left inset) and CRP (right inset) simple axisymmetric model of the BDD process in LSDYNA by means of the finite element method (FEM). This simple model was created to evaluate the BDD operation. The feasibility studies were not validated on experimental deep drawn parts. Nevertheless, the results of a second simulation of cross-shaped cup are compared to experiments later on. In Fig. 5, the constraints and the kinematic sequence are illustrated. Some steps during BDD operation are marked to print corresponding results in Fig. 6. All necessary model parameters are summarized in Table 1 . The elements of the sheet were fully integrated, and isotropic von-Mises yield criterion was used.

The process model was primarily designed to illustrate the local strain hardening effect within a BDD cycle. For this reason, the authors neglected in first order the representation of temperature, velocity-dependent effects and the Bauschinger effect in the material model. It is not yet known how strongly these parameters influence the BDD process. For the demonstrative purpose, material data of common drawing steel was used (see Table 1). A simple piecewise linear plasticity model was implemented to define the hardening.

The development of the effective plastic strain over time increments $1-13$ from Fig. 5 is shown in Fig. 6. In three cycles $(1-5 ; 5-9 ; 9-13)$, the weak zone at the bottom radius is strain-hardened due to alternating bending of the material in the deformation zone. Upsetting is superimposed by the center offset. To cause the steel sheet to be locally upset at the desired position, in the simulation, the die geometry was modified by a bead shape designed specifically for the cylindrical cup. The objective was to produce maximal strain hardening in the desired zone during only a few cycles (dark zone in Fig. 6). Currently, there is no evidence which hardening must be achieved and how many cycles are acceptable.

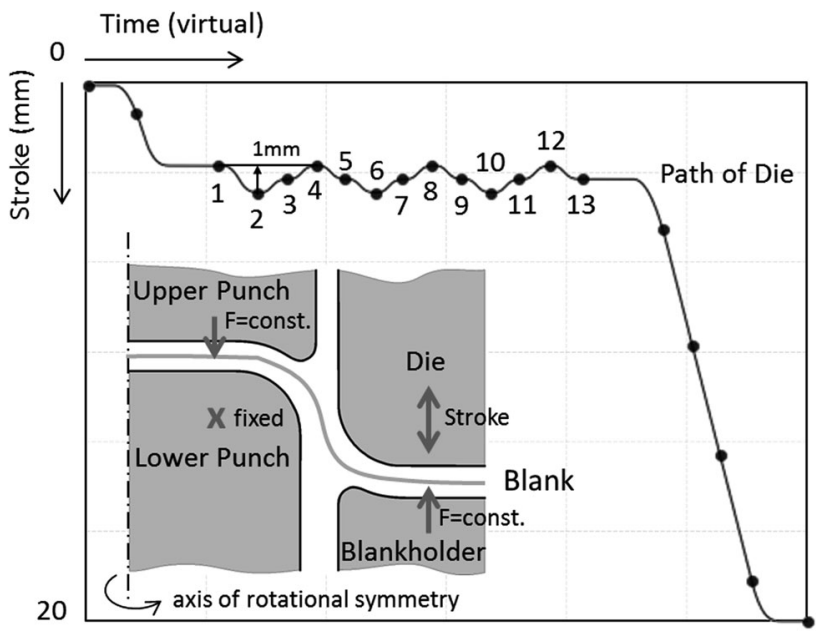

Fig. 5 Principal view of axisymmetric model setup and the pathtime curve 


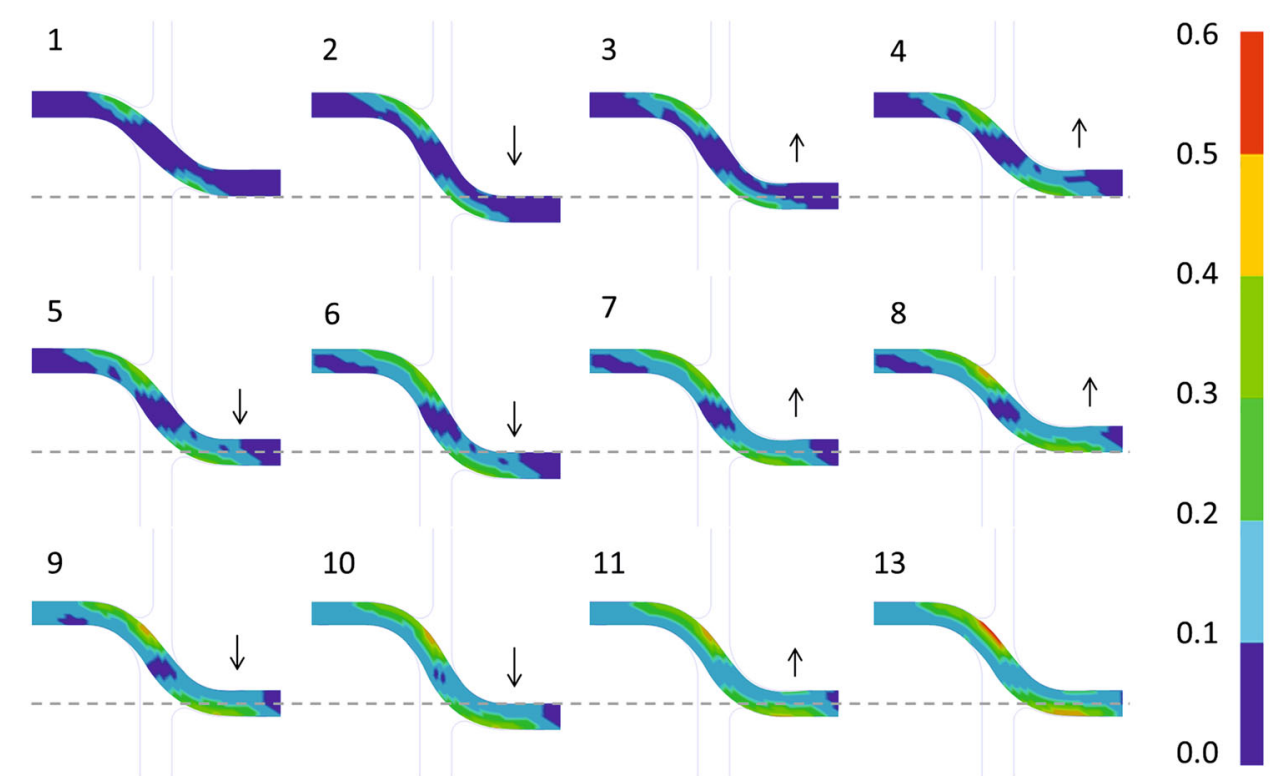

Fig. 6 Sequence of shots showing the development of effective plastic strain during BDD process (the column of color scale indicates the different levels of hardening)

Table 1 Model parameters for the axisymmetric cylindrical cup

\begin{tabular}{ll}
\hline Parameter & Value \\
\hline Sheet material & 1.0338 \\
Sheet thickness & $1 \mathrm{~mm}$ \\
Blank diameter & $100 \mathrm{~mm}$ \\
Lower punch diameter & $50 \mathrm{~mm}$ \\
Lower punch radius & $2 \mathrm{~mm}$ \\
Die radius & $2 \mathrm{~mm}$ \\
Drawing gap & $1.2 \mathrm{~mm}$ \\
Load upper punch & $50 \mathrm{kN}$ \\
Load blankholder & $50 \mathrm{kN}$ \\
Friction coulomb & 0.02 \\
\hline
\end{tabular}

A second simulation gives the chance to compare the simulation to the experiments shown in Fig. 3. Therefore, the authors used the original deep drawing tool, without the bead shape at upper punch and blankholder as shown in Fig. 5. The conventional process model for deep drawing of the cross-shaped cup was created in LS-DYNA. The tools have rigid surfaces, and the boundary conditions were adopted from the experiments (see Fig. 3). Thanks to the double symmetry, the problem was reduced to a quarter of a model. The blank was built with Belytschko-Tsay shells and has five integration points over thickness. In contrast to the preliminary simulations (see Fig. 5; Table 1), another material with higher yield strength was used to extend the hardening effects during few BDD cycles. The material model induces piecewise linear metal plasticity with isotropic von-Mises yield criterion. The material data were obtained from tensile
Table 2 Model parameters for the cross-shaped cup

\begin{tabular}{ll}
\hline Parameter & Value \\
\hline Material & 1.4301 \\
Sheet thickness & $1 \mathrm{~mm}$ \\
Blank size & $340 \mathrm{~mm} \times 300 \mathrm{~mm}$, clipped \\
& edges with $103 \mathrm{~mm}$ at $45^{\circ}$ \\
Punch size & $220 \mathrm{~mm} \times 160 \mathrm{~mm}$ \\
Lower punch radius & $10 \mathrm{~mm}$ \\
Upper punch radius & $10 \mathrm{~mm}$ \\
Die radius & $7.5 \mathrm{~mm}$ \\
Drawing gap & $1.5 \mathrm{~mm}$ \\
Friction coulomb & 0.1 \\
\hline
\end{tabular}

tests. Further parameters are summarized in Table 2. The resulting sheet thinning is illustrated in Fig. 7. The deep drawn part is at the drawing limit, which is defined by critical value of $30 \%$ thinning. As a result of strain hardening performed previously by means of BDD, it is possible to carry out the deep drawing procedure down to a higher depth. An intermediate step at 45-mm drawing depth is demonstrated in Fig. 7. At the necking, the part that experienced BDD is thicker and is thus less damaged by cracking.

The process model is quite simple without any higherorder effects like dynamic oscillations or elastic tools or tilting effects. Material-specific effects have not yet been considered during the forming. In ongoing FE analyses, further kinematic strain hardening has to be taken into account. During alternating tension-compression plasticity in BDD operations, the yield strength decreases each time when the 


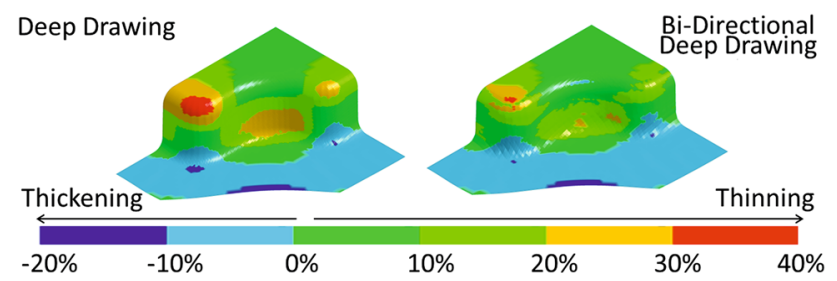

Fig. 7 Thickness reduction at deep drawing limit in comparison for a cross-shaped cup without (left) and with BDD (right)

direction of strain is changed (Bauschinger effect). Also the presumably higher temperature by the alternating bending is not considered. We guess that the current model overestimates the hardening effect during BDD bending. Because of the feasibility calculation, failure parameters could not be validated (e.g., local strain) with measured details on part (e.g., sheet thickness). Nevertheless, both—experiment and simulation—show the increasing drawing depth.

\section{Summary}

This paper introduced a deep drawing variant with alternating bending based on multi-axis kinematic sequences in a state-of-the-art digital multi-axis servo press. The modification of the kinematic sequence can be used to achieve different effects. The approach describes a way to increase the transferable drawing forces over the weak point at the punch radius for high-strength sheets due to a preliminary bi-directional deep drawing process. On the other hand, the opportunity to decrease the required drawing forces for less strain-hardened materials, such as mild steel and some aluminum alloys, by using in-process cushion-ram pulsation is quoted [9]. As a rule, the general goal is to extend the range of constraints in deep drawing: for instance, to obtain higher drawing depth or process reliability. Since the process guidance is complicated, it is necessary to develop software that generates the most productive kinematic press sequence as a function of the technological parameters and the start file for the FE analysis at the same time. Deep drawing procedures with modified kinematic sequences can presumably be controlled based only on FE analyses. There are only slight losses in productivity in the part contact due to the sound dynamic characteristics of state-of-the-art servo presses.

Acknowledgments The presented results of this paper are part of public research projects at Fraunhofer IWU, which is located in Chemnitz, Germany. The Projects 17058BR and 17488BR of the research association "Europäische Forschungsvereinigung für Blechverarbeitung e. V." (EFB) were financed by the "Arbeitsgemeinschaft industrieller Forschungsvereinigungen-Otto von Guericke e. V." (AiF) as part of the program to support "Industrial Community Research and Development" (IGF) with funds from the "Federal Ministry for Economic Affairs and Energy" (BMWi) following an order by the German Federal Parliament. The financial support and co-work are gratefully acknowledged by the authors.

\section{References}

[1] O. Majidi, H. Bong, F. Barlat, M. Lee, C. Kim, Effect of slide velocity on springback in U-draw bending of AHSS sheets with digital servo-press, paper present in Steel Research International, Special Edition: The 14th International Conference on Metal Forming (2012)

[2] J. Landowski, Stamping Journal PMA Publication, pp. 12-14 (2004)

[3] T. Nakano, Press machine trends and servo press forming examples, paper present in 13th International Conference on Metal Forming, Toyohashi (2010)

[4] H. Yamashita, Research in deep-draw forming of high-strength steel sheet using a NC servo press machine, paper present in 50th IDDRG Conference, Graz (2010)

[5] K. Osakada, K. Mori, T. Altan, P. Groche, Mechanical servo press technology for metal forming, paper present in CIRP AnnalsManufacturing Technology, vol. 60 (2011), pp. 651-672

[6] T. Geka, M. Asakura, T. Kiso, T. Sugiyama, M. Takamura, M. Asakawa, Key Eng. Mater. 554-557, 1320 (2013)

[7] H. Hayashi, H. Nishimura, The application of servo press machine to forming of sheet metals with low formability, in The Annals of "DUNAREA DE JOS" University of GALATII, Fascicle V, Technologies in Machine Building, pp. 3-10 (2009)

[8] S. Kaya, Dissertation, Ohio State University (2008)

[9] S. Kriechenbauer, R. Mauermann, P. Müller, Procedia Eng. 81, 905 (2014)

[10] K. Mori, K. Akita, Y. Abe, Int. J. Mach. Tools Manuf 47, 321 (2007)

[11] T. Nakagawa, Servo motor driven press and market trend in Japan, paper present in CIRP 56th General Assembly, Kobe (2006)

[12] Amada Co., Ltd. http://www.amada.co.jp/english/. 21 Feb 2012

[13] K.E.A. Ichikawa, Procedia Eng. 81, 1669 (2014)

[14] I. Komatsu, T. Murakami, Practical use of servo press, in Nikkan-Kougyou-Shinbunsha (2009) 